\title{
Radiative data for Fe XIII
}

\author{
P. R. Young ${ }^{\star}$ \\ Space Science and Technology Department, Rutherford Appleton Laboratory, Chilton, Didcot, Oxfordshire OX11 0QX, UK \\ Received 15 May 2003 / Accepted 23 December 2003

\begin{abstract}
New radiative data for allowed and forbidden transitions between the $3 s^{2} 3 p^{2}, 3 s 3 p^{3}$ and $3 s^{2} 3 p 3 d$ configurations of Fe XIII are presented. The calculations are performed with the SSTRUCT atomic code, including 24 configurations in the model, and term energy corrections have been applied to optimise the theoretical level energies. The new data-set is compared with other calculations in the literature and also an unpublished calculation of Young that is found in the CHIANTI atomic database. Good agreement is found in all cases except with calculations of Nahar. Comparisons are also presented with laboratory and solar experimental data that demonstrate the accuracy of the present calculations. The new data are to be added to version 5 of
\end{abstract} \\ the CHIANTI atomic database.
}

Key words. Sun: general - atomic data - Sun: UV radiation

\section{Introduction}

Fe XIII is a key coronal ion formed in conditions of collisional ionization equilibrium at two million $\mathrm{K}$, typical of the quiet Sun and active regions. It produces a host of lines at extreme ultraviolet wavelengths (Malinovsky \& Heroux 1973; Thomas $\&$ Neupert 1994) due to $3 \mathrm{~s}-3 \mathrm{p}$ and $3 \mathrm{p}-3 \mathrm{~d}$ transitions that provide excellent density diagnostics (Young et al. 1998) over the electron density range $10^{9}-10^{11} \mathrm{~cm}^{-3}$. In addition, transitions within the ground $3 \mathrm{~s}^{2} 3 \mathrm{p}^{2}$ configuration give rise to prominent lines at $\lambda 3389, \lambda 10747$ and $\lambda 10798$ in the optical and nearinfrared. The $\lambda 10747 / \lambda 10798$ ratio is an excellent density diagnostic for ground-based observers (Flower \& Pineau des Forêts 1973; Penn et al. 1994), and is also a potential diagnostic of the coronal magnetic field (Judge 1998).

The CHIANTI atomic database contains a large set of atomic data suitable for the analysis of astrophysical spectra. The current CHIANTI model for Fe XIII is described in Dere et al. (2001) and Dere et al. (1997) and contains radiative data calculated by the author yet not previously published. The model of the ion used in CHIANTI has been improved and the new calculation is presented here and compared both to the older CHIANTI data-set and other calculations. A critical comparison of the collisional data added to v.3 of CHIANTI to that in v.1 has been performed by Landi (2002). The CHIANTI Fe XIII model has been widely used both directly in analyses of spectroscopic data from, e.g., the SOHO satellite, and indirectly through the incorporation of CHIANTI into the TRACE and SOHO/EIT analysis software.

In addition to the CHIANTI model, other Fe XIII models suitable for interpreting solar and stellar spectra have been presented by Brickhouse et al. (1995) and Keenan et al. (1995).

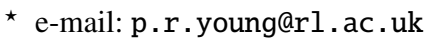

The former work used allowed transition decay rates from Flower \& Nussbaumer (1974) and Fawcett (1987), and forbidden decay rates from Flower \& Pineau des Forêts (1973). Keenan et al. (1995) use radiative decay rates from Mendoza \& Zeippen (1982) and Fawcett (1987).

\section{Calculations and data}

The calculations presented here were performed using the SUPERSTRUCTURE or SSTRUCT code (Eissner et al. 1974). The wave functions are of the configuration-interaction type and are linear combinations of Slater-type orbitals. For Fe XIII, 24 configurations were included and are listed in Table 1. The radial wavefunctions used by SSTRUCT are scaled in a Thomas-Fermi-Dirac statistical model potential, and the scaling parameters were obtained through an optimisation procedure carried out in LS-coupling. The parameter values are given in Table 2 .

The initial stage of the SSTRUCT calculation is the solution of the non-relativistic Hamiltonian, with the addition of one-body and two-body relativistic operators to the Hamiltonian performed subsequently through the method of Saraph (1972). The relativistic wavefunctions can be adversely affected if calculated energy level differences are significantly different from observed level differences for the non-relativistic wavefunctions. One can thus adjust the non-relativistic energies to closer match observed energies (a process called term energy correction, TEC), leading to a modified non-relativistic Hamiltonian. This method has been applied here, and the TECs are given in Table 3 .

Table 4 compares the length and velocity forms of the weighted oscillator strengths for allowed transitions between the LS terms of the $3 \mathrm{~s}^{2} 3 \mathrm{p}^{2}, 3 \mathrm{~s} 3 \mathrm{p}^{3}$ and $3 \mathrm{~s}^{2} 3 \mathrm{p} 3 \mathrm{~d}$ configurations. 
Table 1. Configurations used in the SSTRUCT model.

\begin{tabular}{llll}
\hline \hline Even & & Odd & \\
\hline $3 s^{2} 3 p^{2}$ & $3 s^{2} 3 d^{2}$ & $3 s 3 p^{3}$ & $3 s^{2} 3 p 3 d$ \\
$3 s^{2} 3 p 4 p$ & $3 s^{2} 3 p 4 f$ & $3 s^{2} 3 p 4 s$ & $3 s^{2} 3 p 4 d$ \\
$3 s 3 p^{2} 3 d$ & $3 s 3 p^{2} 4 s$ & $3 s 3 p^{2} 4 p$ & $3 s 3 p^{2} 4 f$ \\
$3 s 3 p^{2} 4 d$ & $3 s^{2} 3 d 4 s$ & $3 s 3 p 3 d^{2}$ & $3 s^{2} 3 d 4 p$ \\
$3 s^{2} 3 d 4 d$ & $3 p^{3} 4 s$ & $3 s^{2} 3 d 4 f$ & $3 p^{3} 3 d$ \\
$3 p^{3} 4 d$ & $3 p^{4}$ & $3 p^{3} 4 p$ & \\
$3 p^{2} 3 d^{2}$ & & & \\
\hline
\end{tabular}

Table 2. Thomas-Fermi-Pauli scaling parameters for the Fe XIII SSTRUCT model.

\begin{tabular}{|c|c|c|c|c|c|c|c|}
\hline \multirow[t]{4}{*}{$1 \mathrm{~s}$} & 1.40089 & $2 s$ & 1.11249 & $3 \mathrm{~s}$ & 1.13553 & $4 s$ & 1.17266 \\
\hline & & $2 p$ & 1.05861 & $3 p$ & 1.10042 & $4 p$ & 1.12312 \\
\hline & & & & $3 d$ & 1.12388 & $4 d$ & 1.14800 \\
\hline & & & & & & $4 f$ & 1.32369 \\
\hline
\end{tabular}

Agreement between the two sets of values gives an indication of the quality of the calculation, and it can be seen that agreement is generally excellent.

Table 5 presents experimental and theoretical energies and percentage compositions for the 27 levels of the three lowest energy configurations, $3 \mathrm{~s}^{2} 3 \mathrm{p}^{2}, 3 \mathrm{~s} 3 \mathrm{p}^{3}$ and $3 \mathrm{~s}^{2} 3 \mathrm{p} 3 \mathrm{~d}$. Following the application of TECs the theoretical energies are within $2 \%$ of the experimental energies. The percentage compositions demonstrate that some levels show substantial mixing due to terms with the same $J$-value and parity mixing through configuration interaction. This leads to decay channels becoming stronger than expected if there is no mixing. For example, level $21\left(3 \mathrm{~s}^{2} 3 \mathrm{p} 3 \mathrm{~d}^{1} \mathrm{D}_{2}\right)$ mixes strongly with level $19\left(3 \mathrm{~s}^{2} 3 \mathrm{p} 3 \mathrm{~d}^{3} \mathrm{P}_{2}\right)$ leading to a large $A$-value for the $21-2$ transition (Table 6), while the $3 \mathrm{~s} 3 \mathrm{p}^{3}{ }^{1} \mathrm{D}_{2}$ level (13) does not mix strongly with the $3 \mathrm{~s} 3 \mathrm{p}^{3}{ }^{3} \mathrm{P}_{2}$ level (12) and so transition $13-2$ has a much smaller $A$-value. Configuration interaction as applied to Fe XIII has been discussed by Nussbaumer (1973).

Table 6 presents wavelengths $(\lambda)$, weighted oscillator strengths $(g f)$ and radiative decay rates $(A)$ for allowed transitions between the $3 \mathrm{~s}^{2} 3 \mathrm{p}^{2}, 3 \mathrm{~s} 3 \mathrm{p}^{3}$ and $3 \mathrm{~s}^{2} 3 \mathrm{p} 3 \mathrm{~d}$ configurations. The wavelengths have been derived from the experimental energy values in Table 5. Table 7 presents $A$-values for forbidden transitions amongst the levels of the ground configuration, and Table 8 gives $A$-values for forbidden transitions that de-populate the metastable level $3 \mathrm{~s}^{2} 3 \mathrm{p} 3 \mathrm{~d}{ }^{3} \mathrm{~F}_{4}$. Note that the two strongest transitions from the ${ }^{3} \mathrm{~F}_{4}$ level are magnetic quadrupole transitions to the ground ${ }^{3} \mathrm{P}_{2}$ and ${ }^{1} \mathrm{D}_{2}$ levels.

\section{Comparison with other data}

There have been a number of publications presenting radiative data for Fe XIII and the most recent are described here. Mendoza \& Zeippen (1982) calculated decay rates for the ground configuration forbidden transitions using SSTRUCT. The model was specifically focussed on the ground forbidden transitions, and included seven configurations of the same parity as $3 \mathrm{~s}^{2} 3 \mathrm{p}^{2}$. Huang (1985) and Kohstall et al. (1998)
Table 3. Term energy corrections applied to the Fe XIII model. The correction is the energy amount added to the theoretical energy to improve agreement to the observed energy.

\begin{tabular}{llc}
\hline \hline Configuration & Term & Correction $\left(\mathrm{cm}^{-1}\right)$ \\
\hline $3 \mathrm{~s}^{2} 3 \mathrm{p}^{2}$ & ${ }^{3} \mathrm{P}$ & 0.0 \\
& ${ }^{1} \mathrm{D}$ & -1951.3 \\
$3 \mathrm{~s} 3 \mathrm{p}^{3}$ & ${ }^{1} \mathrm{~S}$ & -3469.4 \\
& ${ }^{5} \mathrm{~S}$ & +4538.6 \\
& ${ }^{3} \mathrm{D}$ & -84.8 \\
& ${ }^{3} \mathrm{P}$ & -1426.5 \\
& ${ }^{1} \mathrm{D}$ & -3941.0 \\
& ${ }^{3} \mathrm{~S}$ & -7965.3 \\
$3 \mathrm{~s}^{2} 3 \mathrm{p} 3 \mathrm{~d}$ & ${ }^{3} \mathrm{P}$ & -8921.0 \\
& ${ }^{3} \mathrm{~F}$ & -5138.0 \\
& ${ }^{3} \mathrm{P}$ & -9933.7 \\
& ${ }^{1} \mathrm{D}$ & -10425.3 \\
& ${ }^{3} \mathrm{D}$ & -10650.1 \\
& ${ }^{1} \mathrm{~F}$ & -14081.3 \\
& ${ }^{1} \mathrm{P}$ & -12366.1 \\
\hline
\end{tabular}

Table 4. Comparison of length and velocity forms of the weighted oscillator strengths.

\begin{tabular}{lll}
\hline \hline Transition & Length & Velocity \\
\hline $3 \mathrm{~s}^{2} 3 \mathrm{p}^{2}{ }^{3} \mathrm{P}-3 \mathrm{~s} 3 \mathrm{p}^{3}{ }^{3} \mathrm{D}$ & 0.438 & 0.454 \\
$3 \mathrm{~s}^{2} 3 \mathrm{p}^{2}{ }^{3} \mathrm{P}-3 \mathrm{~s} 3 \mathrm{p}^{3}{ }^{3} \mathrm{P}$ & 0.538 & 0.495 \\
$3 \mathrm{~s}^{2} 3 \mathrm{p}^{2}{ }^{3} \mathrm{P}-3 \mathrm{~s} 3 \mathrm{p}^{3}{ }^{3} \mathrm{~S}$ & 1.747 & 1.623 \\
$3 \mathrm{~s}^{2} 3 \mathrm{p}^{2}{ }^{3} \mathrm{P}-3 \mathrm{~s}^{2} 3 \mathrm{p} 3 \mathrm{~d}^{3} \mathrm{P}$ & 2.566 & 2.363 \\
$3 \mathrm{~s}^{2} 3 \mathrm{p}^{2}{ }^{3} \mathrm{P}-3 \mathrm{~s}^{2} 3 \mathrm{p} 3 \mathrm{~d}^{3} \mathrm{D}$ & 6.337 & 6.212 \\
$3 \mathrm{~s}^{2} 3 \mathrm{p}^{2}{ }^{1} \mathrm{D}-3 \mathrm{~s} 3 \mathrm{p}^{3}{ }^{1} \mathrm{D}$ & 0.469 & 0.430 \\
$3 \mathrm{~s}^{2} 3 \mathrm{p}^{2}{ }^{1} \mathrm{D}-3 \mathrm{~s} 3 \mathrm{p}^{3}{ }^{1} \mathrm{P}$ & 0.905 & 0.757 \\
$3 \mathrm{~s}^{2} 3 \mathrm{p}^{2}{ }^{1} \mathrm{D}-3 \mathrm{~s}^{2} 3 \mathrm{p} 3 \mathrm{~d}^{1} \mathrm{D}$ & 2.190 & 2.121 \\
$3 \mathrm{~s}^{2} 3 \mathrm{p}^{2}{ }^{1} \mathrm{D}-3 \mathrm{~s}^{2} 3 \mathrm{p} 3 \mathrm{~d}^{1} \mathrm{~F}$ & 2.914 & 2.895 \\
$3 \mathrm{~s}^{2} 3 \mathrm{p}^{2}{ }^{1} \mathrm{~S}-3 \mathrm{~s} 3 \mathrm{p}^{3}{ }^{1} \mathrm{P}$ & 0.186 & 0.195 \\
$3 \mathrm{~s}^{2} 3 \mathrm{p}^{2}{ }^{1} \mathrm{~S}-3 \mathrm{~s}^{2} 3 \mathrm{p} 3 \mathrm{~d}{ }^{1} \mathrm{P}$ & 1.097 & 1.050 \\
\hline
\end{tabular}

both constructed wavefunctions through the multiconfiguration Dirac-Fock method, where states are represented by a linear combination of configuration state functions, with states divided into groups according to the $J$ value and parity with optimisation performed on each group separately up to $J=2$ (even) and $J=4$ (odd). Huang (1985) used the code of Desclaux (1975), and included only $n=3$ configurations, while Kohstall et al. (1998) used the GRASP92 code (Parpia et al. 1996) and included $n=4$ and $n=5$ configurations in addition to $n=3$. Fawcett (1987) calculated oscillator strengths through optimizing Slater parameters calculated with a Hartree-Fock-Relativistic program package. Three even parity and five odd parity configurations were included, and the Slater parameters were optimised by the Zeeman package through semi-empirical adjustments of the calculated energies. Nahar (1999) performed calculations in the close-coupling approximation with the $R$-matrix method for a large number of transitions, including configurations up to $n=10$. In terms of the $3 s^{2} 3 p^{2}, 3 s 3 p^{3}$ and $3 s^{2} 3 p 3 d$ configurations, only allowed, non-intercombination transition decay rates were presented, 
Table 5. Level indices, experimental and theoretical energy levels, and percentage compositions for the 27 levels of the $3 \mathrm{~s}^{2} 3 \mathrm{p}^{2}, 3 \mathrm{~s} 3 \mathrm{p}^{3}$ and $3 s^{2} 3$ p3d configurations of Fe XIII. Experimental energies are from the NIST database (Martin et al. 1995) except where otherwise stated. The first number in the percentage composition column is the contribution of the identified level. Other contributing levels from the same configuration do not have their configurations identified. Only percentages $\geq 5 \%$ are listed.

\begin{tabular}{|c|c|c|c|c|c|}
\hline \multirow[b]{2}{*}{ Index } & \multirow[b]{2}{*}{ Configuration } & \multirow[b]{2}{*}{ Term } & \multicolumn{2}{|c|}{ Energy $\left(\mathrm{cm}^{-1}\right)$} & \multirow[b]{2}{*}{ Percentage composition of levels } \\
\hline & & & Expt. & Theory & \\
\hline 1 & $3 s^{2} 3 p^{2}$ & ${ }^{3} \mathrm{P}_{0}$ & 0.0 & 0.0 & $93.2 \%$ \\
\hline 2 & & ${ }^{3} \mathrm{P}_{1}$ & $9305.64^{a}$ & 9411.63 & $96.6 \%$ \\
\hline 3 & & ${ }^{3} \mathrm{P}_{2}$ & $18566.76^{a}$ & 18722.40 & $87.7 \%+8.9 \%{ }^{1} \mathrm{D}_{2}$ \\
\hline 4 & & ${ }^{1} \mathrm{D}_{2}$ & 48068 & 48359 & $87.6 \%+8.9 \%{ }^{3} \mathrm{P}_{2}$ \\
\hline 5 & & ${ }^{1} \mathrm{~S}_{0}$ & 91508 & 91753 & $91.8 \%$ \\
\hline 6 & $3 \mathrm{~s} 3 \mathrm{p}^{3}$ & ${ }^{5} \mathrm{~S}_{2}$ & $214624^{b}$ & 214680 & $97.9 \%$ \\
\hline 7 & & ${ }^{3} \mathrm{D}_{1}$ & 287205 & 287237 & $84.0 \%+10.3 \% 3 \mathrm{~s}^{2} 3 \mathrm{p} 3 \mathrm{~d}^{3} \mathrm{D}_{1}$ \\
\hline 8 & & ${ }^{3} \mathrm{D}_{2}$ & 287360 & 287401 & $82.7 \%+9.9 \% 3 \mathrm{~s}^{2} 3 \mathrm{p} 3 \mathrm{~d}^{3} \mathrm{D}_{2}$ \\
\hline 9 & & ${ }^{3} \mathrm{D}_{3}$ & $290180^{b}$ & 290307 & $88.0 \%+10.2 \% 3 \mathrm{~s}^{2} 3 \mathrm{p} 3 \mathrm{~d}^{3} \mathrm{D}_{3}$ \\
\hline 10 & & ${ }^{3} \mathrm{P}_{0}$ & $328925^{b}$ & 329076 & $88.8 \%+9.2 \% 3 \mathrm{~s}^{2} 3 \mathrm{p} 3 \mathrm{~d}^{3} \mathrm{P}_{0}$ \\
\hline 11 & & ${ }^{3} \mathrm{P}_{1}$ & $329706^{b}$ & 329797 & $85.2 \%+9.1 \% 3 \mathrm{~s}^{2} 3 \mathrm{p} 3 \mathrm{~d}^{3} \mathrm{P}_{1}$ \\
\hline 12 & & ${ }^{3} \mathrm{P}_{2}$ & 330279 & 330411 & $77.4 \%+9.0 \% 3 \mathrm{~s}^{2} 3 \mathrm{p} 3 \mathrm{~d}^{3} \mathrm{P}_{2}$ \\
\hline 13 & & ${ }^{1} \mathrm{D}_{2}$ & $362407^{b}$ & 362651 & $52.7 \%+38.7 \% 3 \mathrm{~s}^{2} 3 \mathrm{p} 3 \mathrm{~d}^{1} \mathrm{D}_{2}$ \\
\hline 14 & & ${ }^{3} \mathrm{~S}_{1}$ & 415462 & 415432 & $75.4 \%+18.4 \%{ }^{1} \mathrm{P}_{1}$ \\
\hline 15 & $3 s^{2} 3 p 3 d$ & ${ }^{3} \mathrm{~F}_{2}$ & $432741^{b}$ & 432187 & $96.0 \%$ \\
\hline 16 & & ${ }^{3} \mathrm{~F}_{3}$ & $439420^{b}$ & 439116 & $96.4 \%$ \\
\hline 17 & $3 \mathrm{~s} 3 \mathrm{p}^{3}$ & ${ }^{1} \mathrm{P}_{1}$ & 438050 & 438401 & $68.2 \%+19.6 \%{ }^{3} \mathrm{~S}_{1}+8.7 \% 3 \mathrm{~s}^{2} 3 \mathrm{p} 3 \mathrm{~d}^{1} \mathrm{P}_{1}$ \\
\hline 18 & $3 s^{2} 3 p 3 d$ & ${ }^{3} \mathrm{~F}_{4}$ & 448599 & 449369 & $97.1 \%$ \\
\hline 19 & & ${ }^{3} \mathrm{P}_{2}$ & 486358 & 486456 & $47.3 \%+20.5 \%{ }^{1} \mathrm{D}_{2}+15.5 \% 3 \mathrm{~s} 3 \mathrm{p}^{3}{ }^{1} \mathrm{D}_{2}+7.5 \%{ }^{3} \mathrm{D}_{2}$ \\
\hline 20 & & ${ }^{3} \mathrm{P}_{1}$ & 494942 & 494260 & $58.1 \%+27.6 \%{ }^{3} \mathrm{D}_{1}+6.2 \% 3 \mathrm{~s} 3 \mathrm{p}^{3}{ }^{3} \mathrm{P}_{1}$ \\
\hline 21 & & ${ }^{1} \mathrm{D}_{2}$ & 498870 & 499295 & $34.9 \%+22.2 \%{ }^{3} \mathrm{P}_{2}+23.2 \% 3 \mathrm{~s} 3 \mathrm{p}^{3}{ }^{1} \mathrm{D}_{2}+11.0 \%{ }^{3} \mathrm{D}_{2}$ \\
\hline 22 & & ${ }^{3} \mathrm{P}_{0}$ & $501520^{b}$ & 500393 & $87.2 \%+9.2 \% 3 \mathrm{~s} 3 \mathrm{p}^{3}{ }^{3} \mathrm{P}_{0}$ \\
\hline 23 & & ${ }^{3} \mathrm{D}_{1}$ & 506502 & 506186 & $57.1 \%+28.4 \%{ }^{3} \mathrm{P}_{1}+7.3 \% 3 \mathrm{~s} 3 \mathrm{p}^{3}{ }^{3} \mathrm{D}_{1}$ \\
\hline 24 & & ${ }^{3} \mathrm{D}_{3}$ & 509176 & 509594 & $85.2 \%+9.8 \% 3 \mathrm{~s} 3 \mathrm{p}^{3}{ }^{3} \mathrm{D}_{3}$ \\
\hline 25 & & ${ }^{3} \mathrm{D}_{2}$ & 509250 & 519793 & $67.5 \%+17.1 \%{ }^{3} \mathrm{P}_{2}+8.3 \% 3 \mathrm{~s} 3 \mathrm{p}^{3}{ }^{3} \mathrm{D}_{2}$ \\
\hline 26 & & ${ }^{1} \mathrm{~F}_{3}$ & 556870 & 557043 & $95.4 \%$ \\
\hline 27 & & ${ }^{1} \mathrm{P}_{1}$ & 570690 & 570847 & $85.3 \%+8.9 \% 3 \mathrm{~s} 3 \mathrm{p}^{31} \mathrm{P}_{1}$ \\
\hline
\end{tabular}

${ }^{a}$ Penn \& Kuhn (1994).

${ }^{b}$ Jupén et al. (1993).

and forbidden transitions were not considered. Ishikawa \& Vilkas (2001) present decay rates for the $3 \mathrm{~s}^{2} 3 \mathrm{p}^{2}{ }^{3} \mathrm{P}_{J}-{ }^{3} \mathrm{P}_{J^{\prime}}$ transitions calculated using relativistic multireference many-body perturbation theory. A full set of $n=3$ configurations were included in calculating the state wavefunctions.

For the release of version 1 of the CHIANTI atomic database in 1996, a complete set of radiative data for Fe XIII were calculated by the author using SSTRUCT. This calculation was identical to the one presented here, apart from two differences. The present calculations make use of an updated version of SSTRUCT that corrects a previous bug, and term energy corrections have been performed to improve the accuracy of the theoretical energies (and thus the atomic model). The radiative model currently found in CHIANTI will be referred to as the "CHIANTI" model, while the new calculation presented here will be referred to as the "SSTRUCT" model.

\subsection{Forbidden transitions}

Table 7 compares the forbidden transition probabilities for the ground configuration transitions amongst the Ishikawa \& Vilkas (2001), CHIANTI, Huang (1985), Mendoza \& Zeippen (1982) and present calculations. In general, agreement is excellent, however, the 1-3 decay rate from Ishikawa \& Vilkas (2001) is around a factor 3 lower than from the other three calculations. The length and velocity forms of the E2 decay rates presented by Ishikawa \& Vilkas (2001) are highly discrepant, indicating that their 1-3 decay rate is not accurate. Significant 
Table 6. Observed wavelengths, oscillator strengths and radiative decay rates for allowed transitions amongst the 27 levels of the Fe XIII model.

\begin{tabular}{|c|c|c|c|c|c|c|c|c|c|c|c|c|c|c|}
\hline$j$ & $l$ & $\lambda(\AA)$ & $g f^{a}$ & $A_{j i}\left(\mathrm{~s}^{-1}\right)$ & $j$ & $i$ & $\lambda(\AA)$ & $g f$ & $A_{j i}\left(\mathrm{~s}^{-1}\right)$ & $j$ & & $\lambda(\AA)$ & $g f$ & $A_{j i}\left(\mathrm{~s}^{-1}\right)$ \\
\hline 6 & 2 & 87.05 & $1.02(-3)$ & $5.75(+6)$ & 13 & 3 & 290.83 & $6.95(-3)$ & $1.10(+8)$ & 20 & 4 & 223.78 & $1.78(-2)$ & $7.91(+8)$ \\
\hline 6 & 3 & & & & 13 & 4 & & & & 20 & & 247.87 & & \\
\hline 6 & 4 & 600.40 & & & 14 & 1 & 240.70 & & & 21 & 2 & 204.26 & $6.30(-1)$ & \\
\hline 7 & 1 & 348.18 & $7.47(-2)$ & & 14 & 2 & 246.21 & $4.65(-1)$ & & 21 & 2 & 208.20 & $5.44(-3)$ & \\
\hline 7 & 2 & 9.84 & $2.03(-2)$ & & 14 & 3 & 251.96 & $61(-1)$ & $3.37(+10)$ & 21 & 4 & 221.83 & $1.37(+0)$ & $3.70(+10)$ \\
\hline 7 & 3 & 372.25 & $8.38(-4)$ & $1.34(+7)$ & 14 & 4 & 272.19 & $2.17(-2)$ & $6.52(+8)$ & 22 & 2 & 203.16 & $2.92(-1)$ & $4.71(+10)$ \\
\hline 7 & 4 & 8.17 & $2.44(-3)$ & $3.11(+7)$ & 14 & 5 & 308.69 & $83(-2)$ & $4.27(+8)$ & 23 & 1 & 197.43 & $1.43(-1)$ & +9) \\
\hline 7 & 5 & 0.99 & $3.28(-4)$ & $2.79(+6)$ & 15 & 2 & 236.16 & $8(-3)$ & $9(+8)$ & 23 & 2 & 201.13 & $7.40(-1)$ & $4.07(+10)$ \\
\hline 8 & 2 & 9.64 & $2(-1)$ & $(+9)$ & 15 & 3 & 241.44 & $95(-3)$ & $59(+8)$ & 23 & 3 & 204.95 & $.41(-1)$ & 10) \\
\hline 8 & 3 & 372.03 & $5.02(-3)$ & $4.84(+7)$ & 15 & 4 & 259.96 & $1.84(-2)$ & $3.63(+8)$ & 23 & 4 & 218.13 & $3.24(-2)$ & $1.52(+9)$ \\
\hline 8 & 4 & 7.90 & $1.46(-3)$ & $1.12(+7)$ & 16 & 3 & 237.61 & $2.04(-2)$ & $3.44(+8)$ & 23 & 5 & 240.97 & $316(-1)$ & $1.21(+7)$ \\
\hline 9 & 3 & 368.17 & $1.89(-1)$ & $1.33(+9)$ & 16 & 4 & 255.52 & $145(3)$ & $2.12(+7)$ & 24 & 3 & 203.83 & $82(10)$ & 10) \\
\hline 9 & 4 & 413.03 & & & 17 & 1 & 228.28 & & & 24 & 4 & 216.87 & & $2.47(+9)$ \\
\hline 10 & 2 & & & & 17 & 2 & & & & 25 & 2 & & & \\
\hline 11 & 1 & & & & 17 & 3 & & & & 25 & 3 & & & $3.36(+10)$ \\
\hline 11 & 2 & & & & 17 & 4 & & & & 25 & 4 & 216.83 & & $7.02(+9)$ \\
\hline 11 & 3 & & & & 17 & 5 & 288.57 & & & 26 & 3 & 185.77 & & $3.50(+9)$ \\
\hline 11 & 4 & & & $5.66(+7)$ & 19 & 2 & 209.62 & & $1.85(+10)$ & 26 & 4 & 196.54 & $2.78(+0)$ & $6.86(+10)$ \\
\hline 11 & 5 & & & $2.39(+7)$ & 19 & 3 & 213.77 & & $1.84(+10)$ & 27 & 1 & 175.23 & $4.98(-3)$ & $3.61(+8)$ \\
\hline 12 & 2 & & & & 19 & 4 & 228.16 & & $1.50(+1$ & 27 & 2 & 178.13 & & $1.56(+8)$ \\
\hline 12 & 3 & & & & 20 & 1 & 202.04 & & & 27 & 3 & 181.12 & & $2.70(+6)$ \\
\hline 12 & 4 & & & & 20 & 2 & 205.91 & & $2.61(+8)$ & 27 & 4 & 191.34 & & $2.55(+7)$ \\
\hline 13 & 2 & 283.20 & $4.72(-3)$ & $7.86(+7)$ & 20 & 3 & 209.92 & $1.43(-1)$ & $7.23(+9)$ & 27 & 5 & 208.69 & $1.11(+0)$ & $5.68(+10)$ \\
\hline
\end{tabular}

${ }^{a}$ In this and subsequent tables, $x(y)$ denotes $x \times 10^{y}$.

Table 7. Ground configuration forbidden decay rates. The symbols $\triangleleft$ and $\triangleleft$ indicate that the values are different from those of the present calculations by amounts greater than $30 \%$ and a factor of 2 , respectively.

\begin{tabular}{llllll}
\hline \hline & & Ishikawa \& & & Mendoza \& \\
Transition & SSTRUCT & Vilkas (2001) & CHIANTI & Huang (1985) & Zeippen (1982) \\
\hline $1-2$ & $1.396(+1)$ & $1.385(+1)$ & $1.401(+1)$ & $1.358(+1)$ & $1.39(+1)$ \\
$1-3$ & $6.373(-3)$ & $2.168(-3) \smile$ & $6.348(-3)$ & $6.535(-3)$ & $6.32(-3)$ \\
$1-4$ & $3.247(-3)$ & - & $3.774(-3)$ & $8.589(-3) \longleftarrow$ & $2.55(-3)$ \\
$2-3$ & $9.734(+0)$ & $9.586(+0)$ & $9.867(+0)$ & $1.057(+1)$ & $9.74(+0)$ \\
$2-4$ & $7.173(+1)$ & - & $6.200(+1)$ & $7.548(+1)$ & $7.01(+1)$ \\
$2-5$ & $1.087(+3)$ & - & $9.860(+2)$ & $1.168(+3)$ & $1.07(+3)$ \\
$3-4$ & $8.623(+1)$ & - & $7.557(+1)$ & $9.642(+1)$ & $8.47(+1)$ \\
$3-5$ & $4.540(+0)$ & - & $3.851(+0)$ & $5.019(+0)$ & $4.31(+0)$ \\
$4-5$ & $7.640(+0)$ & - & $7.756(+0)$ & $9.250(+0)$ & $7.60(+0)$ \\
\hline
\end{tabular}

differences are found for the 1-4 transition between the Huang (1985) calculation and the SSTRUCT and Mendoza \& Zeippen (1982) calculations. It is not clear why there is such a large difference in this case, given that otherwise the Huang (1985) calculations show excellent agreement, however, this transition is weak compared to the 2-4 and 3-4 transitions and so plays a negligible role in the level balance of the ion.

The lifetime of the $3 \mathrm{~s}^{2} 3 \mathrm{p}^{2}{ }^{1} \mathrm{D}_{2}$ level has been experimentally determined by Moehs et al. (2001), Träbert et al. (2002) and Träbert et al. (2003), with values of $6.93 \pm 0.18,8.0 \pm 0.1$ and $8.1 \pm 0.2 \mathrm{~ms}$, respectively. The lifetime derived from the SSTRUCT $A$-values is $6.33 \mathrm{~ms}$, which compares with 6.46 and $5.82 \mathrm{~ms}$ from the Mendoza \& Zeippen (1982) and Huang (1985) calculations. Moehs et al. (2001) also presented theoretical calculations from a relativistic multiconfiguration method, yielding a ${ }^{1} \mathrm{D}_{2}$ lifetime of $7.23 \mathrm{~ms}$.

Excellent agreement is found with the Kohstall et al. (1998) calculations for the M1 and E2 transitions that de-populate the metastable ${ }^{3} \mathrm{~F}_{4}$ level (Table 8). However significant differences are found for the magnetic quadrupole transitions 3-18 and 
4-18. The values derived from SSTRUCT were tested by varying the number of configurations included in the model of the ion, as cancellation effects associated with the addition of extra configurations can sometimes lead to large changes in the $A$-values for some transitions. However, for the models considered the changes to the M2 transitions were less than $1 \%$, giving confidence in the SSTRUCT values.

Träbert et al. (2003) have determined a lifetime of $9.0 \pm 0.4 \mathrm{~ms}$ for the metastable ${ }^{3} \mathrm{~F}_{4}$ level which compares with the value $8.56 \mathrm{~ms}$ derived from the present calculations. The Kohstall et al. (1998) calculations yield a lifetime of $9.17 \mathrm{~ms}$.

\subsection{Allowed transitions}

In Fig. 1 the $A$-values for the allowed transitions amongst the $3 \mathrm{~s}^{2} 3 \mathrm{p}^{2}, 3 \mathrm{~s} 3 \mathrm{p}^{3}$ and $3 \mathrm{~s}^{2} 3 \mathrm{p} 3 \mathrm{~d}$ configurations from the Huang (1985), Fawcett (1987), Kohstall et al. (1998) and Nahar (1999) and CHIANTI calculations are compared with the current SSTRUCT model. In each plot the $X$-axis gives the radiative decay rate from Table 6 while the $Y$-axis gives the ratio of the decay rate from the appropriate calculation to that of the present calculation. Good agreement between the two sets of calculations leads to points lying close to 1 . The $Y$-axis is scaled in such a way to reveal greater detail for ratios lying close to 1, but still show the wide range of differences between the calculations (up to 3 orders of magnitude). To determine the accuracy of the different data-sets it is necessary to compare with solar and laboratory data.

For only one level connected to the ground by allowed transitions are there laboratory lifetime data available: $3 \mathrm{~s} 3 \mathrm{p}^{3}{ }^{3} \mathrm{~S}_{1}$ (level 14 in the SSTRUCT model). The predicted lifetime is $17.0 \mathrm{ps}$, while the measured lifetime is $21 \pm 4$ ps (Träbert et al. 1982). The other calculations under consideration yield lifetimes of 15.7 (Huang 1985), 16.8 (Fawcett 1987), 16.5 (Kohstall et al. 1998) and 15.8 ps (Nahar 1999). Thus only the SSTRUCT model value lies within the experimental error bars.

Whereas laboratory lifetime measurements allow the absolute values of radiative decay rates to be checked, solar spectra allow relative decay rates for transitions from the same upper level to be compared. For an optically thin plasma, the branching ratio, $R$, of two lines at wavelengths $\lambda_{1}$ and $\lambda_{2}$ that decay from the same upper level with decay rates $A_{1}$ and $A_{2}$ is $A_{1} \lambda_{2} / A_{2} \lambda_{1}$ (in energy units), and so is independent of the physical conditions of the plasma. The solar corona represents, in most conditions, just such an optically thin environment, and below we consider several observations of Fe XIII branching ratios.

Initial comparisons with Huang (1985) demonstrated large differences with the SSTRUCT model for some transitions. Upon inspection of the data-sets, the reason was found to be due to erroneous level identifications in Huang (1985). The Huang (1985) $3 \mathrm{~s} 3 \mathrm{p}^{3}{ }^{3} \mathrm{~S}_{1}$ and ${ }^{1} \mathrm{P}_{1}$ levels are inverted relative to the SSTRUCT model, while the $3 \mathrm{~s} 3 \mathrm{p}^{3}{ }^{1} \mathrm{D}_{2}, 3 \mathrm{~s}^{2} 3 \mathrm{p} 3 \mathrm{~d}^{1} \mathrm{D}_{2}$, and $3 s^{2} 3 p 3 d{ }^{3} D_{2}$ levels in the Huang (1985) model correspond to the $3 s^{2} 3 p 3 d{ }^{3} D_{2}, 3 s 3 p^{3}{ }^{1} D_{2}$ and $3 s^{2} 3 p 3 d{ }^{1} D_{2}$ levels, respectively, in the SSTRUCT model. Figure 1a shows the
Table 8. Radiative decay rates for forbidden transitions that depopulate the metastable $3 \mathrm{~s}^{2} 3 \mathrm{p} 3 \mathrm{~d}^{3} \mathrm{~F}_{4}$ level. $i$ is the level index of the lower level in the transition. M1 denotes a magnetic dipole transition, M2 a magnetic quadrupole transition, and E2 an electric quadrupole transition. M1+E2 indicates that the M1 and E2 components are comparable in strength. The symbols $\triangleleft$ and $\triangleleft$ indicate that the values are different from those of the present calculations by amounts greater than $30 \%$ and a factor 2 , respectively.

\begin{tabular}{|c|c|c|c|c|}
\hline$i$ & $A_{j i}$ & $\begin{array}{l}\text { Transition } \\
\text { type }\end{array}$ & $\begin{array}{l}\text { Kohstall } \\
\text { et al. (1998) }\end{array}$ & CHIANTI \\
\hline 3 & $2.583(+1)$ & M2 & $9.96(-1) \triangleleft$ & $2.477(+1)$ \\
\hline 4 & $2.222(+1)$ & M2 & $3.97(+1) \triangleleft$ & $2.327(+1)$ \\
\hline 6 & $4.957(+0)$ & E2 & $4.73(+0)$ & $4.309(+0)$ \\
\hline 8 & $1.176(+1)$ & E2 & $1.10(+1)$ & $1.104(+1)$ \\
\hline 9 & $1.449(+1)$ & $\mathrm{M} 1+\mathrm{E} 2$ & $1.41(+1)$ & $1.334(+1)$ \\
\hline 12 & $2.179(+1)$ & E2 & $1.95(+1)$ & $2.195(+1)$ \\
\hline 13 & $2.408(-1)$ & E2 & $2.02(-1)$ & $1.981(-1)$ \\
\hline 16 & $1.552(+1)$ & M1 & $1.87(+1)$ & $1.554(+1)$ \\
\hline
\end{tabular}

comparison with SSTRUCT following these corrections and excellent agreement is found. There are four transitions for which there are differences of more than a factor 2, however none of these transitions have been identified in solar or stellar spectra and so it is not possible to determine which calculation is more accurate.

Several differences between the Nahar (1999) and SSTRUCT data-sets result in changes to predicted emission line strengths that can be compared with measured spectra. In particular, for transitions from level 20, the Nahar (1999) $A$ values imply that the three lines at 202.0, 205.9 and $209.9 \AA$ (decays to levels 1,2 and 3, respectively) are comparable in strength with ratios $\lambda 205.9 / \lambda 202.0$ and $\lambda 209.9 / \lambda 202.0$ of 0.70 and 1.07, respectively. The corresponding SSTRUCT ratios are, however, 0.0055 and 0.15 . The $\lambda 202.0$ line is actually one of the strongest Fe XIII lines, and solar spectra all show it to be much stronger than the $\lambda 209.9$ line: Brosius et al. (1998) give a $\lambda 209.9 / \lambda 202.0$ ratio of $0.19 \pm 0.04$, in excellent agreement with the SSTRUCT model; while, although Malinovsky \& Heroux (1973) do not identify the lines in their spectrum, they are clearly present in their Fig. 1c with a ratio of around 0.2. The $\lambda 205.9$ line is not identified in the Brosius et al. (1998) spectrum implying that it is a weak line, consistent with the SSTRUCT model. The Nahar (1999) $A$ values for these transitions are thus clearly not consistent with observations.

Other problems can be seen for transitions from levels 7 , 8 and 23 . The $\lambda 359.84 / \lambda 348.18$ ratio $(7-2 / 7-1)$ is predicted from the Nahar (1999) data to be 0.66 whereas from the SSTRUCT data it is 0.25 . The measured SERTS-89 ratio is $0.18 \pm 0.04$ (Thomas \& Neupert 1994; Young et al. 1998), much closer to the SSTRUCT value. The Nahar (1999) data predict a $\lambda 372.03 / \lambda 359.64$ ratio $(8-3 / 8-2)$ of 0.29 , while the SSTRUCT data predict a ratio of 0.030 . The $\lambda 359.64$ line is seen in solar spectra, but no emission line is detected at $372.0 \AA$ in high resolution solar spectra (Thomas \& Neupert 1994), consistent with the much smaller ratio found from the SSTRUCT model. The three lines arising from decay of level 23 to the ground ${ }^{3} \mathrm{P}$ levels 

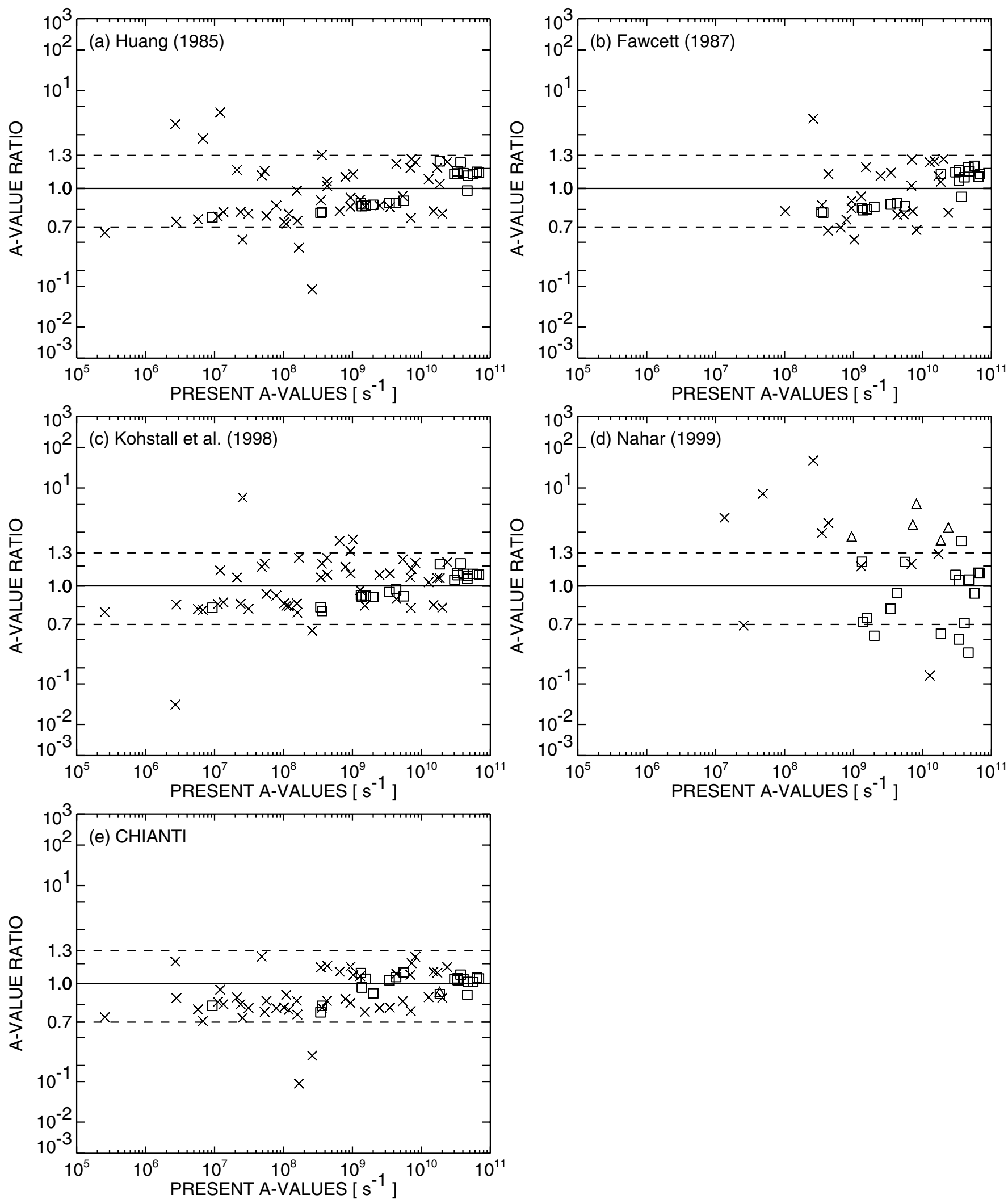

Fig. 1. Plots comparing $A$ values from the present SSTRUCT model to those of a) Huang (1985), b) Fawcett (1987), c) Kohstall et al. (1998), d) Nahar (1999), and e) the CHIANTI model. For each transition for which there exist $A$ values from both calculations, the SSTRUCT $A$ value is plotted against the ratio of the $A$ values from the two calculations. If the transition is the strongest from the particular emitting level in the SSTRUCT model, then the point is plotted with a $\square$. If the transition is not the strongest from the particular emitting level in the SSTRUCT model, but is in the comparative model, then the point is plotted with a $\Delta$. All other transitions are plotted with a $\times$. The $Y$-axis is scaled as the square root of the logarithm of the ratio. Dashed lines are placed at $\pm 30 \%$, while additional minor tickmarks are placed at values 0.9 , $1.1( \pm 10 \%), 0.5,2.0$ (factor 2) and 0.2, 5.0 (factor 5). 
Table 9. Branching ratio comparison. $j$ is the index of the upper emitting level for the ratio.

\begin{tabular}{|c|c|c|c|c|c|c|c|c|c|c|}
\hline \multirow[b]{2}{*}{$j$} & \multirow[b]{2}{*}{ Ratio } & \multicolumn{5}{|c|}{ Theoretical values } & \multicolumn{4}{|c|}{ Observed values } \\
\hline & & $\mathrm{SS}^{a}$ & $\mathrm{CH}^{b}$ & $\mathrm{H} 85^{c}$ & K98 ${ }^{d}$ & $\mathrm{~F} 87^{e}$ & SERTS-89 ${ }^{f}$ & SERTS-95 ${ }^{g}$ & SERTS-9 $97^{h}$ & $\mathrm{MH} 73^{i}$ \\
\hline 7 & $\lambda 359.8 / \lambda 348.2$ & 0.25 & 0.26 & 0.26 & 0.26 & 0.26 & $0.18 \pm 0.04$ & - & - & - \\
\hline 9 & $\lambda 413.0 / \lambda 368.2$ & 0.069 & 0.058 & 0.055 & 0.065 & 0.066 & $0.054 \pm 0.017$ & - & - & - \\
\hline 11 & $\lambda 321.4 / \lambda 312.1$ & 0.46 & 0.50 & 0.48 & 0.50 & 0.48 & $0.38 \pm 0.10$ & - & $0.47 \pm 0.13$ & - \\
\hline 12 & $\lambda 311.6 / \lambda 320.8$ & 0.13 & 0.14 & 0.14 & 0.13 & 0.14 & $0.24 \pm 0.07$ & - & $0.14 \pm 0.07$ & - \\
\hline \multirow[t]{2}{*}{14} & $\lambda 246.2 / \lambda 251.9$ & 0.52 & 0.53 & 0.54 & 0.51 & 0.53 & $0.44 \pm 0.15$ & - & - & 0.60 \\
\hline & $\lambda 240.7 / \lambda 251.9$ & 0.22 & 0.22 & 0.22 & 0.21 & 0.21 & $0.41 \pm 0.18$ & - & - & 0.47 \\
\hline 19 & $\lambda 213.8 / \lambda 209.6$ & 0.98 & 0.99 & 0.82 & 0.94 & 0.72 & $0.62 \pm 0.45$ & $0.44 \pm 0.12$ & - & - \\
\hline 20 & $\lambda 209.9 / \lambda 202.0$ & 0.15 & 0.17 & 0.19 & 0.16 & 0.12 & - & $0.19 \pm 0.04$ & - & - \\
\hline 21 & $\lambda 204.3 / \lambda 221.8$ & 0.59 & 0.55 & 0.43 & 0.47 & 0.74 & $0.93 \pm 0.39$ & - & - & - \\
\hline \multirow[t]{2}{*}{23} & $\lambda 204.9 / \lambda 201.1$ & 0.31 & 0.29 & 0.30 & 0.30 & 0.35 & $0.88 \pm 0.40$ & $0.57 \pm 0.12$ & - & 0.26 \\
\hline & $\lambda 197.4 / \lambda 201.1$ & 0.21 & 0.24 & 0.23 & 0.22 & 0.13 & - & - & - & 0.16 \\
\hline
\end{tabular}

${ }^{a}$ The SSTRUCT model.

${ }^{b}$ The CHIANTI model.

${ }^{c}$ Huang (1985).

${ }^{d}$ Kohstall et al. (1998).

${ }^{e}$ Fawcett (1987).

${ }^{f}$ Thomas \& Neupert (1994).

${ }^{g}$ Brosius et al. (1998). The SERTS-95 spectra were calibrated using atomic data from CHIANTI through insensitive line ratios, including several Fe XIII ratios, and so caution must be used when interpreting these values.

${ }^{h}$ Brosius et al. (2000).

${ }^{i}$ Malinovsky \& Heroux (1973). Intensity errors on individual lines were not explicitly given, but relative intensities are good to $20 \%$ or better.

are found at 197.4, 201.1 and 204.9 $\AA$. The $\lambda 197.4 / \lambda 201.1$ and $\lambda 204.9 / \lambda 201.1$ ratios are predicted to be 0.064 and 1.38 from the Nahar (1999) data, but from SSTRUCT are 0.21 and 0.31, respectively. Malinovsky \& Heroux (1973) give measured ratios of 0.16 and 0.26 , again in much better agreement with the SSTRUCT model.

Nahar (1999) does not include relativistic effects in her calculation, and this would appear to be the source of the differences between this calculation and the other calculations considered here, which all include relativisitic effects.

Excellent agreement is found between the SSTRUCT, Fawcett (1987) and Kohstall et al. (1998) data-sets, with the majority of points in Figs. $1 \mathrm{~b}$ and c lying within $\pm 30 \%$. The one outlying point in Fig. $1 \mathrm{~b}$ is for the 20-2 transition ( $\lambda 205.9)$ which is a factor 3.2 stronger in the Fawcett (1987) calculations, leading to a $\lambda 205.9 / \lambda 202.0$ branching ratio of 0.016 compared to the SSTRUCT value of 0.0055 . The $\lambda 205.9$ line has not been detected in the most sensitive solar spectra in this wavelength region (Brosius et al. 1998), suggesting the SSTRUCT value is more appropriate. Two of the points in the SSTRUCT, Kohstall comparison (Fig. 1c) are more than a factor two discrepant: the 3-27 and 4-27 transitions. These are sufficiently weak transitions that observations can not discriminate which calculation is more suitable, and Fawcett (1987) does not give data for these transitions.
Figure 1e shows a comparison with the radiative data contained in CHIANTI since the version 1 release in 1996. As described earlier in Sect. 2, the model is very similar to the current SSTRUCT model which is revealed in the excellent agreement between the two. Two transitions (2-20 and 3-21) are more than a factor 3 stronger in the new calculation, and we note that these two levels are highly mixed (Table 5) and so the TECs applied in the SSTRUCT calculation will have improved the accuracy of the wavefunctions in this case.

Table 9 compares branching ratios for several line pairs for which there are observed values from solar spectra. This confirms the excellent agreement between the SSTRUCT, CHIANTI, Fawcett (1987) and Kohstall et al. (1998) data-sets, and also demonstrates good agreement with observations.

\section{Summary}

Radiative decay rates for transitions from the Fe XIII $3 s^{2} 3 p^{2}$, $3 \mathrm{~s} 3 \mathrm{p}^{3}$ and $3 \mathrm{~s}^{2} 3 \mathrm{p} 3 \mathrm{~d}$ configurations to the ground $3 \mathrm{~s}^{2} 3 \mathrm{p}^{2}$ configuration have been presented. The data-set includes all allowed and forbidden transitions necessary to model the ion's level populations in low density, coronal conditions.

Comparison has been made with previous calculations for both the allowed and forbidden transitions. Good agreement is found with the forbidden transition calculations of Ishikawa \& Vilkas (2001), Huang (1985) and Mendoza \& Zeippen (1982), and also with the laboratory measurements of the ${ }^{1} \mathrm{D}_{2}$ and ${ }^{3} \mathrm{~F}_{4}$ 
level lifetimes measured by Moehs et al. (2001), Träbert et al. (2002) and Träbert et al. (2003). Good agreement is found for the allowed transitions calculated by Huang (1985), Fawcett (1987) and Kohstall et al. (1998), with many branching ratios involving observed Fe XIII transitions being very similar. Significant differences, however, are found in comparison with the calculations of Nahar (1999). By comparing several branching ratios with measured solar emission line ratios, the latter calculations have been shown to be in error. It is suggested that the differences are due to relativistic effects not being included in the Nahar (1999) calculation.

Several Fe XIII emission lines will be measured by the EUV Imaging Spectrograph (EIS, Culhane et al. 2000) to be flown on the Solar-B satellite (Shimizu 2002). EIS will provide high resolution spectra in the $180-204 \AA$ region which includes the important $\lambda 203.8 / \lambda 202.0$ density diagnostic. The comparisons presented in the present work provide a critical overview of Fe XIII radiative calculations.

Acknowledgements. The author thanks H. E. Mason, E. Landi, G. Del Zanna, and the referee, M. A. Bautista, for useful comments and suggestions.

\section{References}

Brickhouse, N. S., Raymond, J. C., \& Smith, B. W. 1995, ApJS, 97, 551

Brosius, J. W., Davila, J. M., \& Thomas, R. J. 1998, ApJS, 119, 255

Brosius, J. W., Thomas, R. J., Davila, J. M., \& Landi, E. 2000, ApJ, 543,1016

Culhane, J. L., Korendyke, C. M., Watanabe, T., \& Doschek, G. A. 2000, SPIE, 4139, 294

Dere, K. P., Landi, E., Mason, H. E., Monsignori Fossi, B. C., \& Young, P. R., 1997, A\&AS, 125, 149

Dere, K. P., Landi, E., Young, P. R., \& Del Zanna, G. 2001, ApJS, 134, 331
Desclaux, J. P. 1975, Comput. Phys. Commun., 9, 31

Eissner, W., Jones, M., \& Nussbaumer, H. 1974, Comput. Phys. Commun., 8, 270

Fawcett, B. C. 1987, Data Nucl. Data Tables, 36, 129

Flower, D. R., \& Pineau des Forêts, G. 1973, A\&A, 24, 181

Flower, D. R., \& Nussbaumer, H. 1974, A\&A, 31, 353

Huang, K.-N. 1985, Data Nucl. Data Tables, 32, 503

Ishikawa, Y., \& Vilkas, M. J. 2001, Phys. Rev. A, 63, 042506

Judge, P. G. 1998, ApJ, 500, 1009

Jupén, C., Isler, R. C., \& Träbert, E. 1993, MNRAS, 264, 627

Keenan, F. P., Foster, V. J., Drake, J. J., Tayal, S. S., \& Widing, K. G. 1995, ApJ, 453, 906

Kohstall, C., Fritzsche, S., Fricke, B., \& Sepp, W.-D. 1998, Data Nucl. Data Tables, 70, 63

Landi, E. 2002, A\&A, 382, 1106

Malinovsky, M., \& Heroux, L. 1973, ApJ, 181, 1009

Martin, W. C., Sugar, J., Musgrove, A., \& Dalton, G. R. 1995, NIST Database for Atomic Spectroscopy, Version 1.0, NIST Standard Reference Database 61

Mendoza, C., \& Zeippen, C. J. 1982, MNRAS, 199, 1025

Moehs, D. P., Bhatti, M. I., \& Church, D. A. 2001, Phys. Rev. A, 63, 032515

Nahar, S. 1999, Data Nucl. Data Tables, 72, 129

Nussbaumer, H. 1973, A\&A, 27, 303

Parpia, F. A., Froese Fischer, C., \& Grant, I. P. 1996, Comput. Phys. Commun., 94, 249

Penn, M. J., \& Kuhn, J. R. 1994, ApJ, 434, 807

Penn, M. J., Arnaud, J., Mickey, D. L., \& LaBonte, B. J. 1994, ApJ, 436, 368

Saraph, H. E. 1972, Comput. Phys. Commun., 3, 256

Shimizu, T. 2002, Adv. Space Res., 29, 2009

Thomas, R. J., \& Neupert, W. M. 1994, ApJS, 91, 461

Träbert, E., Jones, K. W., Johnson, B. M., Gregory, D. C., \& Kruse, T. H. 1982, Phys. Lett. A, 87, 336

Träbert, E., Gwinner, G., Wolf, A., et al. 2002, J. Phys. B, 35, 671

Träbert, E., Calamai, A. G., Gwinner, G., et al. 2003, J. Phys. B, 36, 1129

Young, P. R., Landi, E., \& Thomas, R. J. 1998, A\&A, 329, 291 